\title{
Percepción subjetiva del esfuerzo en fútbol profesional: relevancia de los indicadores físicos y psicológicos en el entrenamiento y la competición
}

\author{
Antonio J. Gómez-Díaz ${ }^{1,2,}$, Paul S. Bradley ${ }^{3}$, Arturo Díaz y Jesús G. Pallarés ${ }^{4}$ \\ 1 Facultad de Ciencias del Deporte, Universidad de Murcia, Murcia, España \\ 2 Futbol Club Barcelona, Preparador Físico, Segunda División \\ 3 Department of Sport and Exercise Sciences, Soccer Research Group, University of Sunderland, Sunderland, United Kingdom \\ 4 Laboratorio de Fisiología del Ejercicio, Facultad de Ciencias del Deporte, Universidad de Castilla la Mancha, Toledo, España
}

\begin{abstract}
Resumen: Este estudio se llevó a cabo para determinar la relación existente entre la carga física y psicológica de entrenamiento de las sesiones precompetitivas, estimada mediante el tiempo de entrenamiento, la frecuencia cardiaca (FC) y la percepción subjetiva del esfuerzo (PSE), con el propio rendimiento físico de los jugadores en partido oficial. Para ello, en 21 jugadores profesionales de la Football Association Championship Division inglesa, se monitorizó la FC, PSE, el tiempo de entrenamiento y el tiempo específico sobre la zona de alta intensidad en todas las sesiones de entrenamiento durante los 5 días previos al partido. Se registró igualmente la distancia total cubierta y la distancia cubierta en alta intensidad de cada jugador en 22 partidos oficiales mediante el sistema multi-cámara ProZone®. Los resultados indican que los valores reportados de PSE correlacionan con el volumen y la intensidad de entrenamiento desarrollados durante las sesiones preparatorias, y que por lo tanto es una herramienta válida para cuantificar la carga de entrenamiento. No obstante, dentro de las múltiples variables que intervienen en la competición, estos registros de PSE o el promedio de FC durante las sesiones de entrenamiento precompetitivas, no parecen ser variables que permitan predecir el rendimiento condicional de los jugadores en competición oficial.

Palabras clave: Percepción subjetiva del esfuerzo; capacidades físicas; sistema de posicionamiento; deportes de equipo; rendimiento.
\end{abstract}

\section{Introducción}

El estudio sobre los perfiles de esfuerzo y las demandas fisiológicas en los deportes de equipo ha sido especialmente prolífico en las últimas tres décadas, siendo la modalidad deportiva del fútbol la que en mayor medida ha centrado la atención de los investigadores (Bangsbo, Norregaard \& Thorso, 1991; Ekblom, 1986; Mohr, Krustrup \& Bangsbo, 2003). El fútbol es un deporte intermitente, caracterizado por desplazamientos constantes a baja intensidad, combinados con impulsos frecuentes de carrera a máxima velocidad y cortos periodos de recuperación activa (Bradley, Di Mascio, Peart, Olsen \& Sheldon, 2010; Bangsbo, Mohr \& Krustrup, 2006; Bradley, Sheldon, Wooster, Olsen, Boanas \& Krustrup, 2009; Krustrup, Zebis, Jensen \& Mohr, 2010; Mohr, Krustrup, Bangsbo, 2005). Dependiendo del puesto o rol que asuma un futbolista dentro del terreno de juego, diversos estudios han encontrado que cada jugador recorre un total de 10-13 km durante un partido oficial (Bradley et al., 2011; Di Salvo, Gregson, Atkinson, Tordoff \& Drust, 2009; Rampinini, Coutts, Castagna, Sassi, \& Impellizzeri, 2007; Mohr et al, 2003), y que además realizan de media una aceleración máxima o próxima a ser máxima de 10-30 metros cada 90 segundos (Wong, Chamari \& Wisløff, 2010).

* Dirección para correspondencia [Correspondence address]: Antonio José Gómez Díaz. Facultad de Ciencias del Deporte. Campus de San Javier, C/ Argentina s/n. Universidad de Murcia. 30720 Murcia. (España). E-mail: antoniogomez@,institutoinnova.es
Title: Rate of perceived exertion in professional soccer: importance of the physical and psychological factors for training and competition.

Abstract: This study was undertaken to determine the relationship between the pre-competitive physical and psychological training sessions load, estimated by the training time, heart rate (HR) and the rate of perceived exertion (RPE), with the physical performance of players in the competitive match. To do this, in 21 professional players from the English Football Association Championship Division, HR, RPE, the training time and the specific training time in the high intensity zone were monitored during all the training sessions through all the 5 previous days before the match. It was also recorded the total distance covered and the total distance covered in the high intensity zone for each player in 22 official matches using the multi-camera computerised tracking system ProZone ${ }^{\circledR}$. The results indicate that the RPE values correlate with the pre-competitive training volume and intensity, and therefore it is a valid tool to quantify the training load. However, within multiple variables involved in the competition, the RPE or the average HR data recorded during the pre-competitive training sessions do not seem to be variables that can predict the players' conditional performance during the official matches.

Key words: Rate of perceived exertion; fitness; tracking system; team sport; performance.

Los registros de frecuencia cardiaca y el análisis de muestras sanguíneas y musculares obtenidos durante la simulación de partidos de fútbol sugieren que la participación del metabolismo aeróbico es elevada durante todo el encuentro (aproximadamente el 98\%, (Astrand \& Rodhal, 1986)), y que las rutas anaeróbicas de obtención de ATP (Pallarés y Morán-Navarro, 2012), aunque tienen un aporte reducido en el total de la energía requerida, son muy relevantes durante las fases intensas del juego y por lo tanto determinantes del rendimiento deportivo del jugador (Bangsbo, 1994a y 1994b; Ekblom, 1986; Krustrup, Mohr, Steensberg, Bencke, Kjaer \& Bangsbo, 2006; Mohr et al., 2003).

Un adecuado control y manejo de la carga de entrenamiento, entendida como el conjunto de exigencias mecánicas, biológicas y psicológicas que provocan un estado de desequilibrio en el organismo del atleta (González-Badillo \& Serna, 2002), se ha mostrado recientemente crucial para optimizar el rendimiento en futbolistas de alto nivel (Brink, Nederhof, Visscher, Schmikli \& Lemmink, 2010; Jeong, Reilly, Morton, Bae \& Drust., 2011). Entre las principales aplicaciones prácticas de este control y cuantificación de la respuesta interna de cada jugador (i.e., carga interna) se encuentra la posibilidades de individualizar los estímulos de entrenamiento y competición en función de los procesos de recuperación y adaptación, así como una interpretación objetiva sobre los resultados deportivos y de rendimiento alcanzados a medio y largo plazo.

Tanto en la investigación científica como en la práctica deportiva real del fútbol profesional, el método más utiliza- 
do para cuantificar la carga interna del deportista de forma subjetiva ha sido la percepción subjetiva del esfuerzo (PSE) (Borg, 1998), bien sea en su metodología original o con posteriores modificaciones para las que se han descrito índices de validez superiores (Impellizzeri, Rampinini, Coutts, Sassi \& Marcora, 2004; Little \& Williams, 2007). Diferentes autores han reportado la validez de esta herramienta para estimar la carga o fatiga a la que se someten a los deportistas en entrenamiento y competición, siempre entendida como la integración indisoluble de una doble dimensión: física y psicológica (Laurent et al., 2011; Watt and Grove, 1993; Yu, 1998).

Numerosos estudios llevados a cabo en futbolistas de diferentes niveles de rendimiento han observado altos niveles de correlación entre los valores reportados de PSE y otras metodologías de cuantificación de la carga interna por medio de marcadores fisiológicos como la concentración de ácido láctico en sangre capilar (Guner, Kunduracioglu \& Ulkar, 2006), las diferentes rutas metabólicas de obtención de ATP (Casamichana, Castellano, Calleja, Román \& Castagna, 2012) y especialmente la frecuencia cardiaca (FC) (Kelly \& Drust 2009; Mallo \& Navarro, 2009). Estos hallazgos científicos permiten tomar la PSE como un método válido y reproducible para la cuantificación del entrenamiento en deportes colectivos como el fútbol.

La periodización del entrenamiento en el fútbol profesional se realiza atendiendo a multitud de factores tales como el calendario de competiciones y un análisis estratégico de los rivales, el período estacional y el número de partidos pendientes (de Calasanz, García-Martínez, Izquierdo \& García-Pallarés, 2012; Jeong et al., 2011; Reilly \& Ekblom, 2005). Si se tienen en cuenta todos los partidos de liga, copa e internacionales, un jugador profesional participa en más de 60 partidos durante el transcurso de la temporada. La consiguiente carga física y psíquica de este calendario competitivo se ve agravado por la drástica reducción del tiempo de recuperación entre los partidos durante los períodos de alta densidad competitiva (Lago Peñas, Rey, Lago-Ballesteros, Casáis \& Domínguez, 2011; Nieman \& Bishop, 2006; Rowsell, Coutts, Reaburn \& Hill-Haas, 2009). Sin embargo, para el conocimiento de los autores, no existe ningún estudio que haya podido analizar la interconexión y los efectos de los diferentes estímulos de entrenamiento y competición con una adecuada cuantificación de la carga durante las fases de mayor densidad competitiva en futbolistas de alto nivel, que permitan optimizar los procesos de recuperación y maximizar el rendimiento de los futbolistas.

Por todo ello, los objetivos de este estudio fueron: i) detectar la relación existente entre las diferentes variables empleadas comúnmente para realizar la cuantificación de la carga de entrenamiento y competición en un equipo profesional de fútbol a lo largo de una temporada completa, y ii) determinar la relación existente entre las variables psicológicas, las capacidades físicas y sobre el propio rendimiento específico en fútbol. Nuestra hipótesis fue que la PSE es una herramienta válida para cuantificar y controlar tanto la carga de entrenamiento como la de competición en futbolistas profesionales.

\section{Método}

\section{Participantes}

Un total de 21 jugadores varones de fútbol participaron en el estudio. La muestra consistió en jugadores profesionales pertenecientes a clubes de la Football Association Championship Division de la liga inglesa. La media y desviación estándar de las características de los participantes fueron las siguientes: edad $24.8 \pm 3.6$ años, talla $1.85 \pm 0.07 \mathrm{~m}$, la masa corporal $82.4 \pm 6.5 \mathrm{~kg}$ : masa grasa $10.2 \pm 2.2 \%$; experiencia en el entrenamiento de fútbol $14.3 \pm 4.5$ años; experiencia en el fútbol profesional $6.3 \pm 3.4$ años.

\section{Diseño experimental}

Durante 38 semanas se analizaron un total de 190 sesiones de entrenamiento y 22 partidos oficiales pertenecientes al campeonato nacional de Liga Championship Division. En un examen de salud previo no se detectaron limitaciones físicas o lesiones musculo-esqueléticas que pudieran afectar a los sujetos durante la fase experimental, y a todos ellos se les realizó un completo examen médico previo (incluido ECG) descartando cualquier anomalía. El estudio, que cumplió con las directrices de la Asociación Médica Mundial, fue aprobado por su Comité de Bioética y se obtuvo consentimiento informado de todos los sujetos antes de comenzar la investigación.

\section{Cuantificación de la carga de entrenamiento}

Durante las 38 semanas de la temporada competitiva se registraron las variables de PSE, FC, el tiempo total (TT) de entrenamiento y competición, así como el tiempo de esfuerzo específico sobre la zona de alta intensidad (TT $>85 \%$ $\mathrm{FC}_{\max }$ ) en todos los jugadores. Los datos de los jugadores sólo se incluyeron para el análisis final si éstos habían participado en todas las sesiones de preparación semanal, y si se habían registrado las variables que condicionan la cuantificación de la carga durante los partidos. Las unidades detalladas de entrenamiento durante la temporada se basan en las recomendaciones de Bangsbo (2003), donde participan varios microciclos que se extendieron los 7 días previos a los partidos.

La intensidad global de cada sesión de entrenamiento se estableció individualmente de acuerdo a dos variables: a) el porcentaje que suponía la $\mathrm{FC}$ media de la sesión respecto a la FC máxima (\% FC $\left.\mathrm{FC}_{\max }\right)$, y b) la percepción subjetiva del esfuerzo reportada por los jugadores tras finalizar el entrenamiento (PSE). Por su parte, los registros de la FC se tomaron de forma continua en intervalos de $5 \mathrm{~s}$ durante toda la sesión de entrenamiento (Polar, Kempele, Finlandia) siguiendo la metodología de Capranica, Tessitore, Guidetti \& 
Figura (2001). Una vez registrados los valores individuales de la FC, se calculó el valor promedio del ritmo cardíaco en el tiempo total de la sesión (incluyendo el calentamiento, parte principal y vuelta a la calma), así como el tiempo de la sesión que cada jugador realizó esfuerzos de intensidad superior al $85 \%$ de su propia $\mathrm{FC}_{\max }$. Se registró igualmente para cada jugador la estimación de la intensidad global de cada sesión de entrenamiento mediante la PSE (Foster, Hector, Welsh, Schrager, Green \& Snyder, 1995) nada más concluir la vuelta a la calma de cada entrenamiento.

\section{Test incremental en laboratorio}

Todos los participantes realizaron un test incremental en tapiz rodante de laboratorio para determinar, entre otras variables, la frecuencia cardiaca máxima $\left(\mathrm{FC}_{\max }\right)$. Esta valoración se realizó en la segunda semana de la pretemporada y de forma periódica durante la fase competitiva. Todos los jugadores realizaron un calentamiento de 5 min en el tapiz

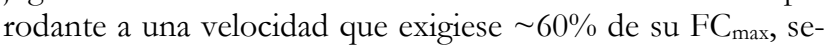
guido de una batería de estiramientos activos del tren inferior. Una vez concluido el calentamiento, la velocidad del tapiz fue incrementándose $1 \mathrm{~km} \cdot \mathrm{h}^{-1}$ cada minuto hasta la extenuación. La frecuencia cardiaca se registró en intervalos de 5 $s$ durante todo el protocolo mediante un monitor de frecuencia cardiaca (Polar Electro Oy, Kempele, Finland). El test se dio por concluido cuando el sujeto solicitó voluntariamente la parada o salió por sus propios medios del tapiz rodante. $\mathrm{La} \mathrm{FC}_{\max }$ se definió como el valor más alto de frecuencia cardiaca registrada durante el test.

\section{Análisis del Partido}

El perfil de actividad de todos los participantes se cuantificó mediante un sistema multi-cámara computerizado de localización (ProZone Sports Ltd ${ }^{\circledR}$, Leeds, Reino Unido) durante los partidos oficiales de competición. La validez y precisión de este sistema de análisis de partido por localización de los jugadores se ha confirmado recientemente en futbolistas de élite (Di Salvo et al., 2007). El perfil de actividad durante los partidos del periodo competitivo se promediaron para reducir la variabilidad (mediana de 3 partidos) (Greg- son, Drust, Atkinson \& Salvo, 2010). En este estudio, la distancia total representa el total de la distancia cubierta durante el partido por el jugador. La distancia en la zona de alta intensidad representa la distancia total cubierta a una velocidad $\geq 19.8 \mathrm{~km} \cdot \mathrm{h}^{-1}$. Estudios previos mostraron que el coeficiente de variación $(\mathrm{CV})$ intra- e inter- observador para esta variables fue $<3 \%$ (Bradley, Sheldon, Wooster, Olsen, Boanas \& Krustrup, 2009).

\section{Análisis estadístico}

Todos los tratamientos estadísticos se realizaron utilizando el programa SPSS para Windows 19.0 (SPSS Inc., Chicago, EE.UU.). La normalidad se verificó mediante las puntuaciones $Z$. Las diferencias entre los registros de los cinco días previos al partido en todas las variables estudiadas fueron analizadas mediante un ANOVA de una vía de medidas repetidas. Una vez detectada una diferencia significativa, la diferencia entre las medias se estableció mediante la prueba post hoc de Bonferroni. Las relaciones entre las variables seleccionadas se evaluaron mediante la correlación de Pearson, con un intervalo de confianza del 95\%. La significación estadística se estableció en $p \leq .05$.

\section{Resultados}

La Tabla 1 muestra la cuantificación de las principales variables que definen el volumen de entrenamiento ( $\mathrm{T}^{\mathrm{T}} \mathrm{T}$ de entrenamiento y T'T $>85 \% \mathrm{FC}_{\max }$ ), así como las variables que permiten estimar la intensidad global requerida por estas sesiones preparatorias (PSE y \% $\mathrm{FC}_{\max }$ ) que llevaron a cabo los jugadores durante los cinco días previos a los partidos oficiales de la temporada. Tanto el TT de entrenamiento, el TT de alta intensidad, así como \% de entrenamiento en alta intensidad del Día -1 fueron estadísticamente inferiores $(p<.05)$ a los días precedentes (i.e., Día -5, Día -4, Día -3 y Día -2). La PSE del Día -3 mostró valores significativamente superiores a los encontrados en el resto de jornadas previas al partido. $\mathrm{El} \% \mathrm{FC}_{\max }$ del Día -1 mostró valores significativamente inferiores $(\phi<.05)$ a los registrados en el resto de jornadas de entrenamiento.

Tabla1. Promedio y desviación estándar del volumen e intensidad de entrenamiento para cada uno de los cinco días previos al partido oficial durante la temporada de competición.

\begin{tabular}{|c|c|c|c|c|c|c|}
\hline & Día -5 & Día -4 & Día -3 & Día -2 & Día -1 & Media semanal \\
\hline TT de entrenamiento (min) & $82.8 \pm 24.5$ & $91.5 \pm 32.8$ & $92.4 \pm 29.2$ & $84.1 \pm 20.8$ & $71.7 \pm 14.5$ & $84.5 \pm 8.3$ \\
\hline $\mathrm{TT}>80 \% \mathrm{FC}_{\max }(\min )$ & $31.9 \pm 12.7$ & $28.3 \pm 17.3$ & $47.4 \pm 17.5$ & $29.9 \pm 11.0$ & $13.1 \pm 9.6$ & $30.1 \pm 12.2$ \\
\hline$\%$ Alta Intensidad (\%) & $39.1 \pm 14.1$ & $30.4 \pm 19.8$ & $46.8 \pm 16.5$ & $34.1 \pm 11.3$ & $16.4 \pm 10.4$ & $33.4 \pm 11.3$ \\
\hline RPE media & $3.6 \pm 1.3$ & $3.8 \pm 2.0$ & $5.3 \pm 2.5$ & $3.7 \pm 1.6$ & $3.1 \pm 1.2$ & $3.92 \pm 0.81$ \\
\hline$\% \mathrm{FC}_{\max }$ media & $77.6 \pm 4.2$ & $75.6 \pm 5.1$ & $77.9 \pm 2.9$ & $76.2 \pm 3.9$ & $70.7 \pm 4.2$ & $75.6 \pm 2.9$ \\
\hline
\end{tabular}

La Figura 1 muestra los resultados obtenidos del análisis de partidos por el sistema multicámara ProZone® llevado a cabo en los partidos oficiales de la temporada. La distancia media recorrida por los jugadores durante los 21 partidos as- cendió a $10631 \pm 568 \mathrm{~m}$ por encuentro, mientras que la distancia cubierta en la zona específica de alta intensidad (velocidad superior a $19.8 \mathrm{~km} \cdot \mathrm{h}^{-1}$ ) promedió los $1166 \pm 112 \mathrm{~m}$. 


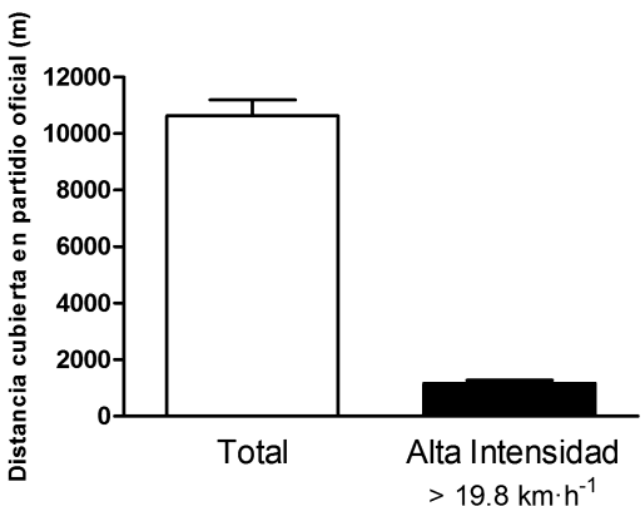

Figura 1. Representación de la distancia total cubierta por los jugadores en partido oficial, así como de la distancia recorrida en la zona de alta intensidad durante la temporada de competición.

La Tabla 2 muestra las correlaciones entre los valores promedio de las principales variables estudiadas durante los días de preparación previos al partido (Días -5 a -1), así como los resultados del análisis del partido con el sistema ProZone ${ }^{\circledR}$ (distancia total cubierta y distancia en la zona de alta intensidad). Cabe destacar las correlaciones significativas que se detectaron al relacionar los valores de PSE reportados por los jugadores y el TT de entrenamiento $(r=.42 ; p<$ $.01)$, el TT $>85 \% \mathrm{FC}_{\max }(r=.41 ; p<.01)$, así como con el $\% \mathrm{FC}_{\max }$ media de entrenamiento $(r=.47 ; p<.01)$. Se detectaron a su vez correlaciones estadísticamente significativas entre TT de entrenamiento y el TT $>85 \% \mathrm{FC}_{\max }(r=.71 ; p$ $<.01)$, al igual que se registraron correlaciones significativas entre la distancia cubierta en partido oficial con la distancia cubierta en la zona de alta intensidad también durante la competición $(r=.71 ; p<.01)$.

No se encontraron, sin embargo, relaciones estadísticamente significativas entre ninguna de las variables que definen el volumen ( $\mathrm{T}^{\circ} \mathrm{T}$ de entrenamiento y $\mathrm{T}^{\prime} \mathrm{T}>85 \% \mathrm{FC}_{\max }$ ) o la intensidad (PSE y \% $\mathrm{FC}_{\max }$ ) de entrenamiento, tanto para los valores medios semanales como para cada uno de los 5 días previos al partido (Días de -5 a -1), con los resultados obtenidos del análisis del partido con el sistema de análisis multi-cámara.

Tabla 2. Correlación entre las principales variables a estudio.

\begin{tabular}{|c|c|c|c|c|c|c|c|}
\hline & 1 & 2 & 3 & 4 & 5 & 6 & 7 \\
\hline 1. TT de entrenamiento & - & $.71^{* *}$ & -.05 & $.42^{* *}$ & -.15 & .29 & .26 \\
\hline 2. $\mathrm{TT}>85 \% \mathrm{FC}_{\max }(\min )$ & - & - & $.65^{* *}$ & $.42^{* *}$ & .23 & -.02 & -.21 \\
\hline 3. $\%$ Alta Intensidad (\%) & - & - & - & .11 & $.47^{* *}$ & -.2 & .38 \\
\hline 4. PSE media & - & - & - & - & .00 & .15 & .31 \\
\hline 5. $\% \mathrm{FC}_{\max }$ media & - & - & - & - & - & -.25 & -.41 \\
\hline $\begin{array}{l}\text { 6. Distancia cubierta en par } \\
\text { tido oficial }\end{array}$ & - & - & - & - & - & - & $.71^{* *}$ \\
\hline $\begin{array}{l}\text { 7. Distancia }>19.8 \mathrm{~km} \cdot \mathrm{h}^{-} \\
\text {en partido oficial }\end{array}$ & 1 & - & - & - & - & - & - \\
\hline
\end{tabular}

\section{Discusión}

Este estudio detalla las relaciones que existen entre los principales componentes de la carga física y psicológica de entrenamiento en las jornadas previas a los partidos oficiales en fútbol profesional, con el propio rendimiento físico del jugador en competición. Los principales hallazgos fueron que la PSE reportada por los jugadores tras las diferentes sesiones preparatorias predice en gran medida el tiempo total de entrenamiento, el volumen de esfuerzo en zonas de alta intensidad, así como la frecuencia cardiaca media. No obstante, la PSE de estas sesiones de entrenamiento que preceden a los partidos de competición en fútbol profesional no parecen condicionar el rendimiento físico que demuestran durante el partido este tipo de futbolistas, expresados en este trabajo como la distancia total recorrida o la distancia cubierta en la zona de alta intensidad. Estos resultados son importantes y únicos, ya que para conocimiento de los autores este el primer estudio que ha podido correlacionar las variables que definen la carga física y psicológica de entrenamiento de futbolistas de alto nivel con su propio rendimiento condicional en partido oficial.

Para este estudio, y a lo largo de toda la temporada de competición, se llevó a cabo una estrategia de tapering o puesta a punto de los partidos oficiales de competición basada en una marcada reducción de la intensidad de la sesión preparatoria (PSE y \% FC $\max$ media) 48 horas antes del partido (Día -2), mientras que el volumen de entrenamiento (TT de entrenamiento y T'T de entrenamiento $>85 \% \mathrm{FC}_{\max }$ ) se mantuvo elevado hasta la jornada previa del encuentro (Día -1, Tabla 1). Esta estrategia de aproximación a la competición en los deportes colectivos basados en un calendario de liga regular se viene empleando en la práctica habitual de la preparación en futbolistas de alto nivel y se ha descrito, con mayor o menor concreción, por diferentes autores $(\mathrm{Mu}-$ jica, 2011; Nikbakht, Keshavarz \& Ebrahim, 2011). Esta propuesta de puesta a punto permite a los jugadores realizar un mantenimiento, o incluso un desarrollo, de las capacidades condicionales básicas, así como un perfeccionamiento de los aspectos técnico-tácticos durante la primera mitad de la semana, mientras que durante las 48 horas previas al partido oficial se prioriza sobre los procesos de recuperación y de optimización del rendimiento integral del jugador.

Los resultados de la cuantificación sobre la distancia total recorrida y la distancia cubierta en la zona de alta intensidad (Figura 1) que se obtienen del análisis del partido oficial con el sistema multi-cámara en el presente estudio (10631 \pm 568 $\mathrm{m})$, son similares a los descritos en estudios recientes donde se reportaron distancias totales cubiertas en partido oficial de futbolistas profesionales de 10-13 km (Bradley et al., 2011; Di Salvo et al, 2009; Mohr et al, 2003; Rampinini et al., 2007), así como las distancias recorridas por los jugadores en la zona de alta intensidad, bien sea en valor absoluto o relativo a la distancia total (Bradley et al., 2011; Bradley et al., 2009; Di Mascio \& Bardley, 2012). 
$\mathrm{Al}$ igual que la frecuencia cardiaca (Bangsbo et al., 2006; Kelly \& Drust 2009; Mallo \& Navarro, 2009) y la concentración de ácido láctico en sangre capilar (Guner et al., 2006), los registros continuados de las precepciones subjetivas del esfuerzo (PSE) se han mostrado como una herramienta válida para cuantificar la carga interna de entrenamiento desde una doble dimensión, física y psicológica, en deportistas de diferentes niveles de rendimiento (Watt and Grove, 1993; Yu, 1998; Impellizzeri et al., 2004). En el caso de la PSE, estos hallazgos son especialmente relevantes ya que se trata de una herramienta idónea para aquellos equipos y grupos de trabajo donde se carezca de los recursos, metodología o el instrumental necesario para monitorizar las variables que definen de forma directa y objetiva la carga interna (p.e., frecuencia cardiaca, concentración de ácido láctico) o externa (p.e., distancia recorrida a diferentes velocidades) del jugador durante todas la sesiones de preparación. Los resultados de este estudio permiten confirmar estos hallazgos, ya que se registraron correlaciones significativas entre los valores PSE reportados por los jugadores y el volumen total de entrenamiento semanal $(r=.42 ; p<.01)$, el tiempo total de entrenamiento en zona de alta intensidad $(r=.41 ; p<.01)$, así como con el $\% \mathrm{FC}_{\max }$ media de entrenamiento $(r=.47 ; p<$ .01). Como principal aplicación práctica, estos resultados sugieren que la PSE permite a los técnicos y preparadores físicos de fútbol cuantificar la carga de entrenamiento a la que han sometido a sus jugadores nada más concluir la sesión preparatoria sin tener que realizar costosos y complejos análisis de los diferentes componentes que definen el volumen y la intensidad de entrenamiento, facilitando y haciéndoles notablemente más accesible esta labor.

No obstante, y refutando una de nuestras hipótesis del estudio, la PSE reportada por los jugadores tras las sesiones preparatorias no parece tener relación alguna con los resultados del rendimiento físico del jugador en el propio partido de competición (i.e., distancia total cubierta y distancia > $19.8 \mathrm{~km} \cdot \mathrm{h}^{-1}$ en partido oficial) (Tabla 2). Tal y como se ha detallado, para el conocimiento de los autores, no existe ningún otro estudio que haya podido relacionar las variables que condicionan la carga de entrenamiento físico semanal con el propio rendimiento específico en partido oficial, por lo que no podemos comparar nuestros resultados. Debido a que el fútbol es un deporte de equipo de rendimiento multifactorial, donde no sólo participan las capacidades físicas y

\section{Referencias}

Astrand, P. O., \& Rodahl, K. (1986). Texbook of work physiology. New York: McGraw-Hill.

Bangsbo, J. (2003). Fitness training in soccer: a scientific approach. Spring City, PA, USA: Reedswain Publishing.

Bangsbo, J. (1994a). Energy demands in competitive soccer. Journal of Sports Sciences, 12 Spec, S5-12.

Bangsbo, J. (1994b). The physiology of soccer--with special reference to intense intermittent exercise. Acta Physiologica Scandinavica, 15 (suppl. 619), $1-155$. psicológicas, sino también las habilidades técnico-tácticas, parece necesario diseñar y llevar a cabo nuevos estudios en este campo que permitan monitorizar las manifestaciones y exigencias técnico-tácticas de las sesiones preparatorias, así como el registro mediante el análisis con multi-cámara en partido oficial de otras variables íntimamente relacionadas con las habilidades y destrezas técnico-tácticas de los jugadores (p.e., la efectividad y precisión en el pase y lanzamientos, desmarques, cruces y robos de balón). A nuestro juicio, un análisis multifactorial de estas características, donde se relacione la carga física, técnico-táctica y psicológica de entrenamiento con el rendimiento específico en competición, permitirá los técnicos y preparadores físicos con recursos limitados poder orientar con mayor probabilidad de éxito sus programas de entrenamiento y de puesta a punto.

\section{Conclusiones}

En resumen, este estudio confirma la validez de la PSE como instrumento para cuantificar la carga de entrenamiento de las sesiones de preparación que constituyen la fase de tapering en futbolistas profesionales, aunque no permite a los técnicos predecir el desempeño físico que tendrán sus jugadores durante el partido a nivel colectivo. Estos resultados sugieren que la PSE se debe tomar como una herramienta fundamental para llevar a cabo la cuantificación de la carga de entrenamiento en fútbol, especialmente en aquellos equipos que carezcan de otros recursos que permiten realizar la cuantificación desde una perspectiva más objetiva con marcadores de carga interna y externa. A pesar de que la PSE permite estimar la carga de trabajo acumulada por los jugadores, los resultados de este estudio indican que, debido a la complejidad y la multitud de factores que condicionan el rendimiento integral del futbolista sobre el terreno de juego, otras variables relacionadas con los aspectos técnico-tácticos deben tenerse en cuenta para poder llegar a predecir el rendimiento específico del jugador durante el partido.

Agradecimientos.- Queremos agradecer la dedicación, el esfuerzo y la profesionalidad de todos los deportistas que tomaron parte en esta investigación, así como la inestimable colaboración del cuadro técnico del club con el registro de todas las variables que se analizaron en este estudio.
Bangsbo, J., Mohr, M. \& Krustrup, P. (2006). Physical and metabolic demands of training and match-play in the elite football player. Journal of Sports Sciences, 24, 665-674.

Bangsbo, J., Norregaard, L. \& Thorso, F. (1991). Activity profile of competition soccer. Canadian Journal of Sports Sciences, 16, 110-116.

Borg, G. (1998). Borg's Perceived Exertion and Pain Scales. Champaign, IL: Human Kinetics.

Bradley, P. S., Carling, C., Archer, D., Roberts, J., Dodds, A., Di Mascio, M., Paul, D., Gomez, A.D. \& Krustrup, P. (2011). The Effect of Playing Formation on High-Intensity Running and Technical Profiles in Eng- 
lish FA Premier League Soccer Matches. Journal of Sports Sciences, 29(8), 821-830.

Bradley, P. S., Di Mascio, M., Peart, D., Olsen, P. \& Sheldon, B. (2010). High-intensity activity profiles of elite soccer players at different performance levels. Journal of Strength \& Conditioning Research, 24, 2343-2351.

Bradley, P. S., Sheldon, W., Wooster, B., Olsen, P., Boanas, P. \& Krustrup, P. (2009). High-intensity running in English FA Premier League soccer matches. Journal of Sports Sciences, 27, 159-168.

Brink, M,S., Nederhof, E., Visscher, C., Schmikli, S.L. \& Lemmink, K.A. (2010). Monitoring load, recovery, and performance in young elite soccer players. Journal of Strength \& Conditioning Research, 24(3), 597-603.

Capranica, L., Tessitore, A., Guidetti, L. \& Figura, F. (2001). Heart rate and match analysis in pre-pubescent soccer players. Journal of Sports Sciences, 19(6), 379-384.

Casamichana, D., Castellano, J., Calleja, J., Román, J.S. \& Castagna, C. (2012). Relationship between indicators of training load in soccer players. Journal of Strength \& Conditioning Research, In Press.

de Calasanz, J., García-Martínez R, Izquierdo, N. \& García-Pallarés, J. (2013). Efectos del entrenamiento de fuerza sobre la resistencia aeróbica y la capacidad de aceleración en jóvenes futbolistas. Journal of Sport \& Health Research, 5(1), 87-94.

Di Salvo, V., Baron, R., Tschan, H., Calderon Montero, F. J, Bachl, N. \& Pigozzi, F. (2007). Performance characteristics according to playing position in elite soccer. International Journal of Sport Medicine, 28(3), 222-227.

Di Salvo, V., Gregson, W., Atkinson, G., Tordoff, P. \& Drust, B. (2009). Analysis of high intensity activity in Premier League soccer. International Journal of Sport Medicine, 30, 205-212.

Di Mascio, M. \& Bradley, P. S. (2012). Evaluation of the most intense highintensity running periods in English FA Premier League soccer matches. Journal of Strength \& Conditioning Research. In Press.

Ekblom, B. (1986). Applied physiology of soccer. Sports Medicine, 3(1), 50-60.

Foster, C., Hector, L. L., Welsh, R., Schrager, M., Green, M. A. \& Snyder, A. C. (1995). European Journal of Applied Physiology \& Occupational Physiology, $70,367-372$.

González-Badillo, J. J. \& Ribas-Serna, J. (2002). Bases de la programación del entrenamiento de la fuerza: Aplicación al alto rendimiento deportivo. (p. 127) Barcelona: INDE.

Gregson, W., Drust, B., Atkinson, G. \& Di Salvo, V. (2010). Match-tomatch variability of high-speed activities in premier league soccer. International Journal of Sports Medicine, 31: 237-242.Guner, R., Kunduracioglu, B. \& Ulkar, B. (2006). Running velocities and heart rates at fixed blood lactate concentrations in young soccer players. Advances in Therapy, 23(3), 395-403.

Impellizzeri, F. M., Rampinini, E., Coutts, A.J., Sassi, A. \& Marcora, S. M. (2004). Use of RPE-based training load in soccer. Medicine \& Science in Sports \& Exercise, 36(6), 1042-1047.

Jeong, T. S, Reilly, T., Morton, J., Bae, S. W. \& Drust, B. (2011). Quantification of the physiological loading of one week of "pre-season" and one week of "in-season" training in professional soccer players. Journal of Sports Sciences, 29(11), 1161-1166.

Kelly, D. M. \& Drust, B. (2008). The effect of pitch dimensions on heart rate responses and technical demands of small-sided soccer games in elite players. Journal of Science \& Medicine in Sport, 12(4), 475-479.

Krustrup, P., Mohr, M., Steensberg, A., Bencke, J., Kjaer, M. \& Bangsbo, J. (2006). Muscle and blood metabolites during a soccer game: implica- tions for sprint performance. Medicine \& Science in Sports \& Exercise, 38, $1165-1174$.

Krustrup, P., Zebis, M., Jensen, J. M. \& Mohr, M. (2010). Game-induced fatigue patterns in elite female soccer. Journal of Strength \& Conditioning Research, 24, 437-441.

Lago-Peñas, C., Rey, E., Lago-Ballesteros, J., Casáis, L. \& Domínguez, E. (2011). The influence of a congested calendar on physical performance in elite soccer. Journal of Strength \& Conditioning Research. 25(8), 21112117.

Laurent, C. M., Green, J. M., Bishop, P. A., Sjökvist, J., Schumacker, R.E., Richardson, M. T. \& Curtner-Smith, M. (2011). A practical approach to monitoring recovery: development of a perceived recovery status scale. Journal of Strength \& Conditioning Research, 25(3), 620-628.

Little, T. \& Williams, A. G. (2007). Measures of exercise intensity during soccer training drills with professional soccer players. Journal of Strength \& Conditioning Research, 21(2), 367-371.

Mallo, J. \& Navarro, E. (2008). Physical load imposed on soccer players during small-sided training games. Journal of Sports Medicine and Physical Fitness, 48(2), 166-171.

Mohr, M., Krustrup, P. \& Bangsbo, J. (2003). Match performance of highstandard soccer players with special reference to development of fatigue. Journal of Sports Sciences, 21, 519-528.

Mohr, M., Krustrup, P. \& Bangsbo, J. (2005). Fatigue in soccer: a brief review. Journal of Sports Sciences, 23, 593-599.

Mujika, I. (2009). Tapering and peaking for optimal performance. Champaign, IL: Human Kinetics.

Nieman, D. C. \& Bishop, N. C. (2006). Nutritional strategies to counter stress to the immune system in athletes, with special reference to football. Journal of Sports Sciences, 24(7), 763-772.

Nikbakht, H., Keshavarz, S. \& Ebrahim, K. (2011). The Effects of Tapering on Repeated Sprint Ability (RSA) and Maximal Aerobic Power in Male Soccer Players. American Journal of Scientific Research, 30, 125-133.

Pallarés, J. G. \& Morán-Navarro, R. (2012). Propuesta metodológica para el entrenamiento de la resistencia cardiorrespiratoria. Journal of Sport and Health Research, 4(2), 119-136.

Rampinini, E., Coutts, A. J., Castagna, C., Sassi, R. \& Impellizzeri, F. M. (2007). Variation in top level soccer match performance. International Journal of Sports Medicine, 28, 1018-1024.

Reilly, T. \& Ekblom, B. (2005). The use of recovery methods post-exercise. Journal of Sports Sciences, 23(6), 619-627.

Rowsell, G. J., Coutts, A. J., Reaburn, P. \& Hill-Haas, S. (2009). Effects of cold-water immersion on physical performance between successive matches in high-performance junior male soccer players. Journal of Sports Sciences, 27(6), 565-573.

Watt, B. \& Grove, R. (1993). Perceived exertion. Antecedents and applications. Sports Medicine, 15(4), 225-241.

Wong, P. L., Chaouachi, A., Chamari, K., Wisløff, U. (2010). Effect of preseason concurrent muscular strength and high-intensity interval training in professional soccer players. Journal of Strength \& Conditioning Research, 24, 653-660.

Yu, M. (1998). Perceived exertion: Integration of psychological and physiological factors. Docotral dissertation, Purdue University.

(Articulo recibido: 18-10-2012; revisado: 25-11-2012; aceptado: 12-12-2012) 